\title{
Experimental status of neutrino scattering physics and needed measurements
}

\section{Tsuyoshi NAKAYA*}

Kyoto University

E-mail: t.nakaya@scphys.kyoto-u.ac.jp

\begin{abstract}
Knowledge of neutrino interactions with a nuclear target at the neutrino energy around sub-GeV is recently getting more and more important as necessary information for accelerator based neutrino oscillation experiments. A study of neutrino interactions contains rich physics of neutrinos with nucleus, and is used to reveal the properties of neutrinos in the oscillation measurements. We review the recent neutrino scattering experiments addressing the physics of neutrino interactions with a nucleus. We list the recent unresolved problems in neutrino interactions, especially modeling of the quasi-elastic scattering process and the neutrino induced signle pion production process. We present the recent status and new results from the $v$-scattering experiments: $\mathrm{K} 2 \mathrm{~K}$, MiniBooNE, MINOS, SciBooNE, T2K and MINERvA. At the end, we summaries the future outlook.
\end{abstract}

10th International Workshop on Neutrino Factories, Super beams and Beta beams

June 30 - July 52008

Valencia, Spain

* Speaker.

${ }^{\dagger}$ for the K2K, Super-K, SciBooNE and T2K collaborations 


\section{Introduction}

After the discovery of neutrino oscillations in atmospheric neutrinos [1], the neutrino mass and mixing are intensively studied by many experiments with solar neutrinos, reactor anti-neutrinos, atmospheric neutrinos and accelerator neutrinos. The study of neutrino oscillation with the accelerator neutrinos is one of the most promising approaches to reveal the unknown properties of neutrinos, a whole scheme of the neutrino mixing and the particle and anti-particle $(\mathrm{CP})$ asymmetry in neutrinos, and to provide precise information on the neutrino mass. A next generation accelerator neutrino oscillation experiment, T2K [2], attracts many attentions to provide the new information. At the same time, we recognize that the further precision studies in the accelerator neutrino oscillation experiments request more accurate information on interactions of neutrinos with a nuclear target which is the target material of massive neutrino detectors for neutrino detection. From the view point, recently, many attentions are drew to understand and improve the knowledge of neutrino interactions with a nuclear target at the energy around sub-GeV. In order to address these issues, several neutrino scattering experiments are conducted to study the neutrino interactions with a nuclear target. The neutrino scattering experiments are introduced in Section 3.

The charged-current (CC) quasi-elastic (QE) scattering with a nuclear target is the primary channel in neutrino interactions which must be well-understood and properly modeled because it is a dominant process for sub-GeV neutrinos and is often used to reconstruct the neutrino energy in experiments. Although it looks like a straight-forward process with a nucleon of which many data exists, complicated phenomena are involved by treating a nuclear target. It is explained in Section 2 and many recent experiments are focused on understanding of the CC-QE process.

The single pion $(1 \pi)$ production by neutrino interactions is also the most important channel in neutrino interactions because it is a dominant process for sub-GeV neutrinos and it is a severe background in neutrino oscillation experiments. A search for $v_{\mu} \rightarrow v_{e}$ oscillation is one of the most interesting subjects in future experiments, and the neutral-current (NC) $1 \pi^{0}$ production is the background in $\mathrm{K} 2 \mathrm{~K}$ and $\mathrm{T} 2 \mathrm{~K}[2,3]$. So, it is important to understand the NC- $1 \pi^{0}$ process and to measure the cross section itself and the momenta of the final produced $\pi^{0}$ which potentially suffers with the effects of nuclear medium. For a precise study of neutrino oscillation in the $v_{\mu} \rightarrow v_{\mu}$ oscillation channel, a dominant background is the $\mathrm{CC}-1 \pi$ process where we miss the pion either by nuclear effects or by the detection technique. In addition to study neutrino oscillation, this channel is equally important to search for a proton-decay, $p \rightarrow e^{+}\left(\mu^{+}\right) \pi^{0}$, because the CC- $1 \pi^{0}$ is a dominant background by atmospheric neutrinos $[4,5]$. We know that the understanding of this process is important to develop a next generation proton decay experiment with a Megaton-size Water Cherenkov detector [6].

Finally, the experimental data of anti-neutrino interactions around sub-GeV are not rich and no data exists on the $\mathrm{CC}-\mathrm{QE}$ process below $1 \mathrm{GeV}$. The future $\mathrm{CP}$ violation study on neutrino oscillations in the accelerator experiments [7] requests equal knowledge of neutrinos and anti-neutrino interactions where the probability of $v_{\mu} \rightarrow v_{e}$ oscillation is compared with that of $\bar{v}_{\mu} \rightarrow \bar{v}_{e}$ after carefully subtraction of the background processes. The anti-neutrino interactions must be wellunderstood with the same level as neutrino interactions although it has lower cross sections. In addition, an anti-neutrino beam in low energy has typically a contamination of neutrinos, which make the background understanding somehow complicated. Currently, the MiniBooND and Sci- 
BooNE experiments described in Section 3 collect anti-neutrino data and under analysis.

\section{Puzzles}

When modern neutrino oscillation experiments, K2K and MiniBooNE, started, it was found that our knowledge of neutrino interactions was not well satisfied to describe the experimental data. For the simulation of neutrino interactions, the K2K collaboration used the NEUT program library $[8,9]$ and the MiniBooNE collaboration used the NUANCE [10]. Both NEUT and NUANCE adopt the similar theoretical models with the nuclear effects, and it is not a clear guide which simulator is better to describe the data. Both K2K and MiniBooNE collaborations intensively studied the neutrino interactions, and found some hints why the experimental data do not match well with the MC prediction. Here, we introduce the unforeseen results in the CC-QE scattering and the single pion production.

\subsection{Quasi-Elastic scattering}

The CC-QE process is thought to be well described in the vector and axial-vector form factors which are expressed with the dipole formula. The vector form factor is well known by data of electron scattering experiments. The axial-vector form factor with the dipole formula has only one parameter, called $M_{A}$, and the $M_{A}$ was measured by many past experiments. The mean value of $M_{A}$ is $1.03 \mathrm{GeV} / \mathrm{c}^{2}$ with the error of a few percent. The observed $Q^{2}$ distributions in $\mathrm{K} 2 \mathrm{~K}$ and MiniBooNE tend to be more energetic than the MC model prediction with $M_{A}=1.03$. The measurements of $\mathrm{K} 2 \mathrm{~K}$ with a water target is $M_{A}=1.20 \pm 0.12 \mathrm{GeV} / \mathrm{c}^{2}[11]$ and the measurement of MiniBooNE with a carbon target is $M_{A}^{e f f}=1.23 \pm 0.20 \mathrm{GeV} / \mathrm{c}^{2}$ [12]. Here, the MiniBooNE collaboration defines a new interpretation for $M_{A}$ as an effective $M_{A}$ because our observation is not a scattering with a nucleon, but with a nuclear target. Therefore, the observation might be suffered by the nuclear effect. Furthermore, the MiniBooNE collaboration also introduces the Pauli-suppression parameter, $\kappa=1.019 \pm 0.011$. It is very interesting to test the CC-QE modeling proposed by the MiniBooNE collaboration by upcoming new experiments.

\subsection{Single pion production}

As introduced in Section 1, the single pion production is the second dominant channel at the sub-GeV neutrino energy. One interesting subject is again the form factors of this process because even the simpler model of CC-QE is claimed to modify the axial form factor for the nuclear target as described in Section 2.1. It looks natural that the similar effect could be observed in the CC- $1 \pi$ channel as CC-QE. However, the existing data for the CC- $1 \pi$ process is very limited and the clear extraction of signal is not as easy as for CCQE. Therefore, we have no conclusion yet.

Another interesting subject is the process of coherent pion production by neutrinos. The K2K experiments reported the null result of the CC coherent $\pi$ production [13] although the clear observation was expected by the Rein and Sehgal model [14]. Rein and Sehgal updated their models by including the lepton mass effect [15]. Recently the MiniBooNE collaboration finds the evidence of $\mathrm{NC}$ coherent $\pi^{0}$ production [16]. It is interesting to check the consistency between the null $\mathrm{K} 2 \mathrm{~K}$ 
result on CC coherent $\pi$ and the positive signal of NC coherent $\pi^{0}$ together by comparing with several theoretical models. Recently, the SciBooNE collaboration looks for the signal of CC coherent $\pi$ production [17].

Finally, another most important quantity is the momentum distribution of pions because it directly affects the background level in neutrino oscillation experiments. So far, K2K and MiniBooNE measured the $\pi^{0}$ momentum distribution in NC- $1 \pi^{0}$ production $[16,18]$. The observed $\pi^{0}$ momentum looks softer than the MC predictions, which may indicate that the treatment of the nuclear effects is not good enough. For future neutrino oscillation experiments, the better estimation of the pion momentum distributions by the MC simulations are necessary to accurately predict the background. To improve the prediction, further experimental data are important to resolve the issues.

\subsection{Low energy electron excess}

The last puzzle in neutrino interactions is the low energy elecron excess observed by MiniBooNE [19]. This phenomena is only observed by the MiniBooNE experiment because MiniBooNE has the best sensitivity to identifying $v_{e}$ in the muon neutrino beam. Although the MiniBooNE collaboration intensively investigates the source of the events, the origin of the excess is not identified yet. Although the impact of this background to $\mathrm{T} 2 \mathrm{~K}$ was concerned, it may be marginal because the sensitivity of $\mathrm{T} 2 \mathrm{~K}$ around $0.4 \%$ in $v_{e}$ appearance is not severe to take matter of this background by comparing with the sensitivity of MiniBooNE around $0.1 \%$.

\section{Neutrino Scattering Experiments}

Physics with neutrino scattering is conducted by using the detectors in neutrino oscillation experiments and a few dedicated neutrino scattering experiments. The former case is the neutrino detectors located close to the neutrino beam source in the K2K, MINOS and T2K experiments and the detector in MiniBooNE. The latter case is the SciBooNE and MINERvA experiments. The experiments are summarized in Table 1 for the mean neutrino beam energy $\left.\left(<E_{v}\right\rangle\right)$, the detector type, the fiducial mass of the detector, and the target material and the number of neutrino events interacted in the detector (\#v events). The "\# $\nu$ " events is shown as a guide to know roughly how many events are occurred in the detector with the full running time of the experiment. Those experiments cover the neutrino energy from a few hundred $\mathrm{MeV}$ up to $30 \mathrm{GeV}$ with various nuclear targets: $\mathrm{H}_{2} \mathrm{O}, \mathrm{CH}_{2}, \mathrm{CH}, \mathrm{Fe}, \mathrm{Pb}, \mathrm{C}$. In addition, several different detector technologies are used for the studies.

\subsection{K2K}

$\mathrm{K} 2 \mathrm{~K}$ is the first generation long-baseline neutrino oscillation experiments from KEK to Kamioka. The K2K neutrino beam was produced by the KEK 12-GeV Proton Synchrotron and had the mean energy of $1.3 \mathrm{GeV}$ for neutrinos. $\mathrm{K} 2 \mathrm{~K}$ has the near and far detectors, and the far detector is SuperKamiokande. The K2K near detector system consisted of 1 kiloton Water Cherenkov detector and sets of the fine-grained detectors (SciFi, SciBar) with the electro-magnetic calorimeter (EC) and the muon range detector (MRD). The K2K experiment and the near detectors are well described in 
Table 1: Summary of Neutrino Scattering Experiments

\begin{tabular}{|c|c|c|c|c|c|}
\hline & $\begin{array}{c}<E_{v}> \\
(\mathrm{GeV})\end{array}$ & Detector & $\begin{array}{c}\text { Fiducial mass } \\
\text { (ton) }\end{array}$ & $\begin{array}{c}\text { Target } \\
\text { Target }\end{array}$ & $\begin{array}{c}\text { \#v events } \\
\text { (as reference) }\end{array}$ \\
\hline K2K (Near) & $\sim 1.3$ & Water Cherenkov, FGD & $5 \sim 50$ & $\mathrm{H}_{2} \mathrm{O}, \mathrm{CH}, \mathrm{Fe}$ & $50 k \sim 100 k$ \\
\hline MiniBooNE & $\sim 0.7$ & Oil Cherenkov & $\sim 440$ & $\mathrm{CH}_{2}$ & $\sim 1 M$ \\
\hline MINOS (Near) & $2 \sim 30$ & Iron/Plastic sandwich & 33 & $\mathrm{Fe}$ & $\sim 5 M$ \\
\hline SciBooNE & $\sim 0.7$ & FGD & 10 & $\mathrm{CH}$ & $\sim 100 k$ \\
\hline T2K (Near) & $\sim 0.7$ & FGD & $2 \sim 10$ & $\mathrm{Fe}, \mathrm{Pb}, \mathrm{CH}, \mathrm{H}_{2} \mathrm{O}$ & $\sim 500 k$ \\
\hline MINER $v \mathrm{~A}$ & $2 \sim 30$ & FGD & $\sim 3$ & $\mathrm{Fe}, \mathrm{Pb}, \mathrm{C}$ & $\sim 10 M$ \\
\hline
\end{tabular}

the reference [20]. The K2K near detectors located inside KEK have rich data on neutrino scattering, and provided several important results on the neutrino scattering physics $[5,11,13,18,21]$. In addition to the published results, $\mathrm{K} 2 \mathrm{~K}$ recently shows the preliminary results on the CC-QE $M_{A}$ with a carbon target [22] and the CC single $\pi^{0}$ production [23] by using the SciBar detector [24]. The results on the CC-QE $M_{A}$ has the value of $M_{A}=1.144 \pm 0.077$ ( fit $)_{-0.072}^{+0.078}$ (syst .) higher by comparing with the mean value of old experiments. The new value of $M_{A}$ is consistent with the former result of K2K with the water target [11] and the result of MiniBooNE with the carbon target [12]. The result on the cross section of the CC single $\pi^{0}$ production is found to be slightly higher than the prediction by models implemented in NEUT $[8,9]$ by $(39 \pm 15) \%$. Those new results are under preparation for publication.

\subsection{MiniBooNE}

MiniBooNE is a short-baseline neutrino oscillation experiment to test the evidence of neutrino transition claimed by LSND. MiniBooNE reported the result not supporting the LSND signal [19]. The MiniBooNE detector is a mineral oil Cherenkov detector of $\sim 1,000$ tons with 1,520 PMTs [25]. MiniBooNE has very high statistics data of neutrino interactions with 450 ton fiducial mass. MiniBooNE collected data of $6.6 \times 10^{20}$ POT (Protons-On-Target) with a neutrino beam configuration and $2.5 \times 10^{20}$ POT with an anti-neutrino beam. By using $5.6 \times 10^{20}$ POT neutrino beam data, MiniBooNE measured the CC-QE scattering on Carbon with an effective axial mass of $M_{A}^{e f f}=1.23 \pm 0.20 \mathrm{GeV} / \mathrm{c}^{2}$ [12]. In addition, MiniBooNE find a deficit of low $Q^{2}$ events which is accounted by introducing the Pauli-suppression parameter, $\kappa=1.019 \pm 0.011$. MiniBooNE detected 28,600 $\pi^{0}$ events and find an evidence of $\mathrm{NC}$ coherent $\pi^{0}$ production [16]. With an advantage of high statistics data, the MiniBooNE collaboration extends various physics analysis including the anti-neutrino interactions.

\subsection{MINOS}

MINOS is the long-baseline neutrino oscillation experiment to confirm the neutrino oscillation and to measure neutrino oscillation parameters precisely. The NuMI (Neutrinos at the Main Injector) neutrino beam for MINOS has the tunability for the neutrino energy and flux, and is typically 
operated with the low energy configuration which produces the neutrinos at the peak energy of $3 \sim 4 \mathrm{GeV}$ and with the flux tail extending up to $30 \mathrm{GeV}$. The MINOS experiments collected data of more than $4 \times 10^{20}$ POT. The MINOS front detector (FD) is located $1 \mathrm{~km}$ away from the target and has the total mass of 1 kilo-ton. The detector is a sandwich type of magnetized iron and plastic scintillators with the magnetic field of $1.2 \mathrm{~T}$. Because of the high intensity NuMI neutrino beam, the MINOS FD collected 5,500,000 CC neutrino interactions in the fiducial mass of 33 tons with $3 \times 10^{20}$ POT. The MINOS collaboration can study the CCQE scattering, a low energy neutrino flux extraction, an inclusive CC cross-section shape, DIS and structure function, and CC and NC coherent pion production although they have not get the preliminary results yet. MINOS expects to have 800,000 CC-QE events at $3 \times 10^{20}$ POT, 2,600,000 $v$-DIS events and 300,000 $\bar{v}$-DIS events at $7.4 \times 10^{20}$ POT, $1,700 \mathrm{NC}$ coherent $\pi^{0}$ events and $2,700 \mathrm{CC}$ coherent $\pi^{+}$events at $3 \times 10^{20}$ POT.

\subsection{SciBooNE}

SciBooNE is a dedicated neutrino scattering experiment to study neutrino interactions physics at FNAL-BNB [26]. The SciBooNE beam is FNAL-BNB and the detector located $100 \mathrm{~m}$ downstream from the proton target. The SciBooNE detector is the K2K SciBar detector [24] and Electron-Catcher (EC) together with the newly built muon range detector. SciBooNE collected data of $0.99 \times 10^{20}$ POT with a neutrino beam configuration and $1.54 \times 10^{20}$ POT with an anti-neutrino beam. The first results from SciBooNE were presented in this conference [27, 28]. The SciBooNE collaboration studies the $\mathrm{CC}-\mathrm{QE}$ process, $\mathrm{CC}-1 \pi$ process, $\mathrm{NC}-1 \pi^{0}$ process, and $\mathrm{NC}$ elastic process with neutrino beam data. The SciBooNE collaboration search for the CC-coherent $\pi$ process and the preliminary result was presented at ICHEP2008 [17]. The analysis of anti-neutrino beam data is on-going and is expected to be available in 2009.

\subsection{T2K}

$\mathrm{T} 2 \mathrm{~K}$ is the next generation long-baseline neutrino oscillation experiments from Tokai to Kamioka. The T2K neutrino beam will be produced by the J-PARC MR accelerator with the initial energy of $30 \mathrm{GeV}$. The narrow-band neutrino beam with the mean energy of $0.7 \mathrm{GeV}$ is produced by the off-axis technique with the off-axis angle of 2.5 degree. The far detector is Super-Kamiokande, and the near detector consists of so-called neutrino beam monitor (named INGRID) and off-axis neutrino detector (ND280-offaxis) located $280 \mathrm{~m}$ downstream from the target. INGRID is a modular type sandwich detector of iron and plastic scintillators. INGRID consists of 16 modules with 10 ton each. The primary purpose of INGRID is not to study the neutrino interactions, but to monitor the neutrino beam. For this purpose, experimental inputs of the neutrino interactions with iron is important for INGRID. The ND280-offaxis is the fine grained tracking detectors mounted inside of the UA1 dipole magnet with the magnetic field of $0.2 \mathrm{~T}$. The ND280-offaxis consists of a dedicated $\pi^{0}$ detector (P0D), two fine-grained scintillator vertex detectors (FGD) with a water target, three large-volume TPCs sitting side by side of FGDs, Electron-Calorimeter (ECAL), and a side muon range detector (SMRD). The uniqueness of T2K ND280-offaxis is the finer granuality of $1 \times 1 \mathrm{~cm}^{2}$ scintillators, momentum measurement with the magnetic field and fine-segmented ECAL. Therefore, it is expected to provide the most precise information on neutrino interactions once it starts the operation. The first T2K beam is expected to be shot in April 2009. The installation of ND280 is 
scheduled in summer 2009 and data taking will start around the end of 2009. So, in the near future, we expect more rich data on neutrino interactions for a precision neutrino oscillation experiment.

\subsection{MINER $v$ A}

MINERvA is a high precision neutrino scattering experiment at the FNAL Main Injector. The MINERvA will use the NuMI neutrino beam. The detector will locate in front of the MINOS front detector. The MINER $v$ A detector is a finely segmented, fully active, scintillator tracking chamber, surrounded by Electro-magnetic and Hadron calorimeters. The uniqueness of MINER $v \mathrm{~A}$ is a wide variety of nuclear targets to measure the $A$ dependence of neutrino interactions. The target nuclei are $\mathrm{He}, \mathrm{C}, \mathrm{Fe}$, and $\mathrm{Pb}$. They plan to run for four years with the NuMI low energy beam configuration for $4 \times 10^{20}$ POT and the medium energy configuration for $12 \times 10^{20}$ POT. With a typical beam exposure, it is expected to collect $\sim 14$ Million neutrino interactions which are used to study the CC-QE process, the resonance meson production, the transition region of the resonance dominance to DIS, the DIS low $Q^{2}$ region, the structure functions, the coherent pion production and the charm and strange production. The experiment is planed to finish installation and commissioning in 2009, and to start taking data in 2010.

\section{Summary and Outlook}

The experimental aspects of neutrino scattering physics are reviewed in this paper. Although the neutrino scattering physics are getting more and more important, there is no special virtue to resolve the current situation of poor understanding and no perfect modeling of many neutrino interaction processes with a nuclear target. It is a only way to settle the puzzles one by one by improving the quality of experimental data. Since several modern experiments are attacking these puzzles to improve the sensitivity of neutrino oscillations, we are looking forward to the breakthrough of neutrino scattering physics in the near future.

\section{Acknowledgment}

The author would like to acknowledge support by Ministry of Education, Culture, Sports, Science and Technology in Japan (MEXT) and Japan Society for the Promotion of Science (JSPS) with the Grant-in-Aid for Scientific Research A 19204026, Young Scientists S 20674004, Scientific Research on Priority Areas "New Developments of Flavor Physics", and the global COE program "The Next Generation of Physics, Spun from Universality and Emergence".

\section{References}

[1] Y. Fukuda et al. [Super-Kamiokande Collaboration], Phys. Rev. Lett. 81, 1562 (1998) [arXiv:hep-ex/9807003].

[2] Y. Itow et al. [The T2K Collaboration], arXiv:hep-ex/0106019.

[3] S. Yamamoto et al. [K2K Collaboration], Phys. Rev. Lett. 96, 181801 (2006) [arXiv:hep-ex/0603004].

[4] M. Shiozawa et al. [Super-Kamiokande Collaboration], Phys. Rev. Lett. 81, 3319 (1998) [arXiv:hep-ex/9806014]. 
[5] S. Mine et al. [K2K Collaboration], Phys. Rev. D 77, 032003 (2008) [arXiv:0801.0182 [hep-ex]].

[6] T. Nakaya, Nucl. Phys. Proc. Suppl. 138 (2005) 376.

[7] T. Nakaya, Prepared for 4th Workshop on Neutrino Oscillations and their Origin (NOON2003), Kanazawa, Japan, 10-14 Feb 2003

[8] Y. Hayato, Nucl. Phys. Proc. Suppl. 112, 171 (2002).

[9] G. Mitsuka, AIP Conf. Proc. 981, 262 (2008).

[10] D. Casper, Nucl. Phys. Proc. Suppl. 112, 161 (2002) [arXiv:hep-ph/0208030].

[11] R. Gran et al. [K2K Collaboration], Phys. Rev. D 74, 052002 (2006) [arXiv:hep-ex/0603034].

[12] A. A. Aguilar-Arevalo et al. [MiniBooNE Collaboration], Phys. Rev. Lett. 100, 032301 (2008) [arXiv:0706.0926 [hep-ex]].

[13] M. Hasegawa et al. [K2K Collaboration], Phys. Rev. Lett. 95, 252301 (2005) [arXiv:hep-ex/0506008].

[14] D. Rein and L. M. Sehgal, Nucl. Phys. B 223, 29 (1983).

[15] D. Rein and L. M. Sehgal, Phys. Lett. B 657, 207 (2007) [arXiv:hep-ph/0606185].

[16] A. A. Aguilar-Arevalo et al. [MiniBooNE Collaboration], Phys. Lett. B 664, 41 (2008) [arXiv:0803.3423 [hep-ex]].

[17] K. Hiraide [SciBooNE Collaboration], arXiv:0810.3903 [hep-ex].

[18] S. Nakayama et al. [K2K Collaboration], Phys. Lett. B 619, 255 (2005) [arXiv:hep-ex/0408134].

[19] A. A. Aguilar-Arevalo et al. [The MiniBooNE Collaboration], Phys. Rev. Lett. 98, 231801 (2007) [arXiv:0704.1500 [hep-ex]].

[20] M. H. Ahn et al. [K2K Collaboration], Phys. Rev. D 74, 072003 (2006) [arXiv:hep-ex/0606032].

[21] A. Rodriguez et al. [K2K Collaboration], Phys. Rev. D 78, 032003 (2008) [arXiv:0805.0186 [hep-ex]].

[22] X. Espinal and F. Sanchez, AIP Conf. Proc. 967 (2007) 117.

[23] C. Mariani [K2K Collaboration], AIP Conf. Proc. 967, 174 (2007).

[24] K. Nitta et al., Nucl. Instrum. Meth. A 535, 147 (2004) [arXiv:hep-ex/0406023].

[25] A. A. Aguilar-Arevalo et al. [MiniBooNE Collaboration], arXiv:0806.4201 [hep-ex].

[26] A. A. Aguilar-Arevalo et al. [SciBooNE Collaboration], arXiv:hep-ex/0601022.

[27] J. L. Alcaraz, Experimental studies of low energy charged current neutrino interactions, in proceedings of 10th International Workshop on Neutrino Factories, Super beams and Beta beams (NuFact08), June 30 - July 5 2008, Valencia, Spain PoS (NUFACT 08 ) 062.

[28] H. Tanaka, Experimental studies of neutral current pion production at low energy, in proceedings of 10th International Workshop on Neutrino Factories, Super beams and Beta beams (NuFact08), June 30 - July 5 2008, Valencia, Spain PoS (NUFACT08) 063. 